\title{
Avaliação audiológica em pacientes com doenças reumáticas pediátricas
}

\section{Hearing loss assessment in patients with pediatric rheumatic disorders}

\section{Carolina Ferreira Campos-Flumian', Jair Cortez Montovani², Claudia Saad Magalhães ${ }^{1}$}

\section{RESUMO}

Objetivo: Realizar avaliação audiológica em crianças e adolescentes acompanhados em um ambulatório de referência para doenças autoimunes, independentemente do diagnóstico específico. Métodos: Foi realizado um estudo cego simples do tipo caso-controle. Foram incluídos 48 pacientes com idades entre 5 e 19 anos e tempo de seguimento de um a 151 meses, divididos em três grupos: 15 pacientes controle com diagnóstico de dor em membros e exclusão de doença autoimune, 23 pacientes com Artrite Idiopática Juvenil, e dez pacientes com outras doenças autoimunes. Os voluntários foram submetidos a avaliações clínica, otológica e audiológica (timpanometria, pesquisa dos reflexos acústicos, audiometria, índice de reconhecimento de fala, emissões otoacústicas e potenciais evocados auditivos de tronco encefálico com estímulo click). Resultados: O grupo com outras doenças autoimunes teve maior número de pacientes sintomáticos e maior número de orelhas alteradas no teste de emissões otoacústicas em comparação com o grupo controle e com o grupo com Artrite Idiopática Juvenil. Ainda no grupo com outras doenças autoimunes, 50\% dos sujeitos assintomáticos apresentaram alterações na pesquisa dos reflexos, na audiometria e nas emissões otoacústicas. Na audiometria, o grupo com Artrite Idiopática Juvenil apresentou mais alterações nas frequências altas, e o grupo com outras doenças autoimunes, nas frequências baixas. Conclusão: Houve maior número de sintomas relacionados à perda auditiva e a alterações audiológicas em crianças e adolescentes com Artrite Idiopática Juvenil e outras doenças autoimunes. As alterações auditivas ocorreram também em pacientes assintomáticos, justificando-se a avaliação audiológica como rotina clínica desses pacientes.

Descritores: Autoimunidade; Audiologia; Deficiência auditiva; Doenças reumáticas; Pediatria

\begin{abstract}
Purpose: To perform audiological assessment in children and adolescents followed up at a reference outpatient clinic for autoimmune diseases, regardless of specific diagnoses. Methods: A single-blind case-control study was conducted. Participants were 48 patients with ages from 5 to 19 years and one to 151 months follow-up, categorized into three groups: 15 control individuals with pain in limbs and no autoimmune disorders, 23 individuals with Juvenile Idiopathic Arthritis, and ten cases diagnosed with other autoimmune disorders. All subjects were submitted to clinical, otological, and audiological assessments (tympanometry, acoustic reflex, audiometry, speech audiometry, otoacoustic emissions, and auditory brainstem response test with click stimuli). Results: The group with other autoimmune disorders had a greater proportion of patients with symptoms and more altered results in the otoacoustic emission test, when compared with the control group and the group with Juvenile Idiopathic Arthritis. In the group with other autoimmune disorders, $50 \%$ of the subjects with no symptoms presented impaired acoustic reflexes, alterations in audiometry and in otoacoustic emissions. In the audiometry, the group with Juvenile Idiopathic Arthritis presented more alterations in higher frequencies, and the group with other autoimmune disorders, in lower frequencies. Conclusion: Symptoms related to hearing loss and audiological alterations were more frequent in children and adolescents with Juvenile Idiopathic Arthritis and other autoimmune disorders. The hearing alterations also occurred in patients with no symptoms, indicating the need for systematic hearing assessment for these patients in their clinical routine.
\end{abstract}

Keywords: Autoimmunity; Audiology; Hearing loss; Rheumatic diseases; Pediatrics

Estudo realizado no Departamento de Pediatria, Faculdade de Medicina de Botucatu, Universidade Estadual Paulista "Júlio de Mesquita Filho" - UNESP Botucatu (SP), Brasil.

(1) Departamento de Pediatria, Faculdade de Medicina de Botucatu, Universidade Estadual Paulista "Júlio de Mesquita Filho" - UNESP - Botucatu (SP), Brasil. (2) Departamento de Otorrinolaringologia, Faculdade de Medicina de Botucatu, Universidade Estadual Paulista "Júlio de Mesquita Filho" - UNESP - Botucatu (SP), Brasil.

Conflito de interesses: Não

Contribuição dos autores: $C F C F$ e $C S M$ participaram do projeto, coleta de dados, análise, interpretação dos resultados e escrita do artigo. JCM participou da avaliação e interpretação dos testes auditivos. Todos os autores leram e aprovaram o manuscrito.

Endereço para correspondência: Claudia Saad Magalhães. Departamento de Pediatria, Faculdade de Medicina de Botucatu, Universidade Estadual Paulista UNESP, Distrito de Rubião Junior, s/n, Botucatu (SP), Brasil, CEP: 18618-970. E-mail: claudi@ fmb.unesp.br

Recebido em: 2/6/2012; Aceito em: 2/4/2013 


\section{INTRODUÇÃO}

Há evidências de perda da acuidade auditiva ou da capacidade de discriminação sonora, zumbido e vertigem em adultos com doenças reumáticas auto-imunes ${ }^{(1-10)}$. Mas, os estudos pediátricos são escassos, dada a menor prevalência destas doenças em crianças e adolescentes ${ }^{(11-14)}$.

$\mathrm{Na}$ disacusia neurossensorial auto-imune como apresentação isolada, relatada tanto no adulto como na criança, o exame clínico, audiológico, e otoscopia são normais, havendo perda auditiva neurossensorial, geralmente assimétrica, bilateral, progressiva ou súbita. Os principais achados histopatológicos na orelha interna foram degeneração de células do gânglio espiral, atrofia do órgão de Corti, atrofia da estria vascular, perda das células ciliadas e perda do labirinto membranoso ${ }^{(15)}$. O tratamento precoce com prednisona pode evitar a progressão da perda auditiva ou até mesmo revertê-la, sendo considerada entre os poucos casos de perda auditiva neurossensorial reversível ${ }^{(2)}$. Pacientes com doenças reumáticas auto-imunes normalmente respondem bem ao tratamento com prednisona ${ }^{(16)}$.

Questiona-se também se as doenças auto-imunes sistêmicas poderiam ter envolvimento auditivo semelhante. As doenças reumáticas auto-imunes são caracterizadas principalmente pelo acometimento estrutural e/ou funcional, de múltiplos órgãos e sistemas, que ocorre devido à inflamação, podendo causar dano permanente ${ }^{(1,2,17)}$. Por apresentarem envolvimento multissistêmico, evolução e prognóstico variáveis, faz-se necessária a monitorização de manifestações pouco sintomáticas, ou aquelas onde a faixa etária limita a discriminação como no caso das manifestações oculares e auditivas.

Existem poucos relatos na literatura sobre avaliação auditiva em doenças auto-imunes pediátricas. Mediante a sua baixa prevalência e, sobretudo, pela importância do comprometimento auditivo para o desenvolvimento infantil, objetivou-se investigar sistematicamente a audição destes pacientes em um serviço especializado em Reumatologia Pediátrica.

\section{MÉTODOS}

Foi realizado um estudo do tipo caso-controle cego-simples, onde os pacientes com doenças reumáticas pediátricas foram submetidos sistematicamente à avaliação clínica e otológica-audiológica independentes. O protocolo foi aprovado pelo Comitê de Ética em Pesquisa da Faculdade de Medicina de Botucatu, Universidade Estadual Paulista "Júlio de Mesquita Filho" (UNESP) (no 374/2004) e o Termo de Consentimento Livre e Esclarecido foi assinado pelos pais ou representantes legais.

Foram critérios de inclusão a idade entre 2 e 19 anos, seguimento no serviço de pelo menos duas consultas e o diagnóstico confirmado por um especialista. Os critérios de exclusão foram a presença de sintomas como febre, dor, mal estar durante os testes, diagnóstico concomitante de doenças genéticas ou malformações associadas, doenças neurológicas como encefalopatia crônica progressiva ou não progressiva, otoscopia alterada na data da avaliação, deficiência auditiva congênita ou pós-infecção, otite média crônica ou colesteatomatosa e surdez familial.

Não houve seleção de casos, foram incluídos pacientes voluntários e consecutivos em uma amostra de conveniência, cujos pais consentiram por meio da assinatura do termo de consentimento. Após a conclusão de todas as avaliações independentes por um dos autores (CFCF), mediante ao diagnóstico revelado por outro autor (CSM), os sujeitos foram divididos em três grupos:

- Grupo controle: casos de crianças saudáveis com dor músculo-esquelética de origem não inflamatória ${ }^{(18)}$.

- Grupo Artrite Idiopática Juvenil (AIJ): casos de Artrite Idiopática Juvenil, diagnosticados de acordo com o critério de classificação da International League of Associations for Reumatology (ILAR) $)^{(19)}$.

- Grupo outras doenças auto-imunes (ODAI): casos de Lúpus Eritematoso Sistêmico Juvenil (LESJ), Esclerodermia Localizada Juvenil, síndrome de Sjögren Primária, sendo todos os casos diagnosticados de acordo com os critérios clínicos estabelecidos ${ }^{(17,20-22)}$.

Foram incluídos 48 sujeitos com idades entre cinco e 19 anos, sendo 15 do grupo controle, 23 com Artrite Idiopática Juvenil e dez com outras doenças auto-imunes. Os diagnósticos específicos são apresentados na Tabela 1.

Tabela 1. Frequência do diagnóstico específico nas doenças reumáticas

\begin{tabular}{lc}
\hline Principais Diagnósticos & Frequência \\
\hline Artrite Idiopática Juvenil (AIJ) & 23 \\
Oligoarticular & 13 \\
Poliarticular & 4 \\
Sistêmica & 4 \\
Artrite relacionada à entesite & 1 \\
Artrite psoriásica & 1 \\
Outras doenças auto-imunes (ODAI) & 10 \\
Lúpus eritematoso sistêmico juvenil & 4 \\
Esclerodermia localizada juvenil & 4 \\
Síndrome de Sjögren juvenil & 2 \\
\hline
\end{tabular}

Todos os testes audiológicos foram realizados pelo mesmo avaliador/autor (CFCF). As avaliações otológicas incluíram um inquérito completo de sintomas auditivos e os exames clínicos foram realizados por um Otorrinolaringologista/autor (JCM).

\section{Avaliação audiológica}

A avaliação audiológica foi composta por: imitanciometria, incluindo a timpanometria e pesquisa dos reflexos acústicos; audiometria; índice de reconhecimento de fala; emissões otoacústicas (EOA) e avaliação dos potenciais evocados auditivos 
de tronco encefálico (PEATE) com estímulo click.

O Imitanciômetro utilizado foi o Amplaid ${ }^{\circledR} 775$ com sonda de $226 \mathrm{~Hz}$. As curvas timpanométricas foram classificadas de acordo com Jerger (1970) ${ }^{(23)}$ (tipos A, Ar, Ad, B e C).

A pesquisa do reflexo acústico foi realizada nas frequências de 500, 1000, 2000 e $4000 \mathrm{~Hz}$, sendo considerado normal o reflexo desencadeado com intensidade entre 70 e 90 dBNA acima do limiar auditivo.

Foi realizada audiometria tonal liminar ou condicionada, de acordo com a idade do paciente. Como instrumento foi utilizado o audiômetro Amplaid ${ }^{\circledR}$ A321 de 2 canais; e fone de ouvido TDH 49P para avaliar a via aérea nas frequências de 250 a $8000 \mathrm{~Hz}$. A via óssea foi avaliada nas frequências de 250 a $4000 \mathrm{~Hz}$ utilizando-se um vibrador ósseo B-71. Foram considerados como perda auditiva os resultados maiores que $15 \mathrm{~dB}$ na via aérea e maiores que 10 na via óssea ${ }^{(24)}$.

A audiometria vocal foi avaliada por meio do teste IRF (índice de reconhecimento de fala) e a porcentagem de acertos maior ou igual a $92 \%$ foi considerada dentro da normalidade ${ }^{(25)}$.

Foi realizado o exame de Emissões Otoacústicas Evocadas Transientes (EOAT) sendo utilizado como estímulo o click, o qual abrange um espectro de frequência de 1000 a $4000 \mathrm{~Hz}$, com intensidade entre 75 e 85 dBNPS. O aparelho utilizado foi o ILO-92 da Otodinamic $®$, na função ILO88 acoplado a um computador. As emissões foram consideradas presentes, segundo os padrões previamente estabelecidos no serviço: reprodutibilidade maior que $50 \%$, estabilidade da sonda maior que $75 \%$, relação sinal/ruído maior que $6 \mathrm{dBNPS}$ em pelo menos três frequências.

Os Potenciais Evocados Auditivos de Tronco Encefálico (PEATE) de curta latência foram obtidos utilizando-se o equipamento Neuropack2 da Nihon Kohden ${ }^{\circledR}$, o qual estava devidamente aterrado para minimizar as interferências eletromagnéticas. Foi utilizado como parâmetro: estímulo click na intensidade de $85 \mathrm{dBnHL}$ com mascaramento contralateral de $-40 \mathrm{~dB}$, polaridade rarefeita, tempo de análise de $10 \mathrm{~ms}$, apresentação de 1000 cliques. A duplicação de cada registro foi realizada para assegurar a reprodutibilidade e fidedignidade das ondas. Os traçados do PEATE de cada paciente foram analisados e interpretados levando-se em consideração a presença de ondas I, III e V, morfologia do traçado e amplitude das ondas. Os valores de latência considerados dentro do padrão de normalidade foram: Pico da onda I (PI) de 1,3 a 1,8 ms; (PIII) de 3,5 a 4,0 ms; (PV) de 5,2 a 5,9 ms. Latência interpicos (LI-III) de 1,8 a 2,3 ms; (LIII-V) de 1,7 a 2,2 ms (LI-V) de 3,7 a 4,5 ms. Foram consideradas dentro dos padrões de normalidade a diferença inter-aural da onda $\mathrm{V}<0,4 \mathrm{~ms}$.

\section{Análise estatística}

A apresentação dos dados demográficos e clínicos foi por meio de estatística descritiva. A comparação dos resultados dos testes audiológicos entre pacientes e controles foi por meio do teste t de Student, análise de variância (ANOVA), ou KruskalWallis seguido do teste de comparações múltiplas de Dunn. A associação entre as variáveis categóricas foi pelo teste do Qui-quadrado ou pelo teste Exato de Fisher.

\section{RESULTADOS}

\section{Dados clínicos e audiológicos}

As características clínicas, imunológicas e o tratamento dos pacientes (grupos AIJ e ODAI) são demonstradas na Tabela 2. Houve diferença significativa $\left(\mathrm{p}=0,02 ; \mathrm{x}^{2}=5,0516\right)$ entre os dois grupos no que se refere ao uso de antiinflamatório não hormonal (AINH). Foi observado também que a maior parte dos pacientes (61\% do AIJ e $80 \%$ do ODAI) estava em fase de atividade da doença e usando medicação no dia da avaliação audiológica.

Tabela 2. Aspectos clínicos, imunológicos e terapêuticos dos pacientes com doenças reumáticas pediátricas na data da avaliação audiológica

\begin{tabular}{lcc}
\hline \multirow{2}{*}{ Variáveis } & $\begin{array}{c}\text { Grupo com AIJ } \\
(\mathrm{n}=23)\end{array}$ & $\begin{array}{c}\text { Grupo com ODAI } \\
(\mathrm{n}=10)\end{array}$ \\
\cline { 2 - 3 } & $\mathrm{x} \pm \mathrm{DP}$ & $\mathrm{x} \pm \mathrm{DP}$ \\
\hline Tempo de seguimento (meses) & $37,9 \pm 39,6$ & $30,1 \pm 34,4$ \\
\hline Fase ativa da doença & $\mathrm{n}(\%)$ & $\mathrm{n}(\%)$ \\
Auto-anticorpos & $14(61)$ & $8(80)$ \\
Anticorpo antinuclear positivo & $6(26)$ & $5(50)$ \\
Fator reumatóide positivo & 6 & 4 \\
Medicamentos & 2 & 2 \\
Antiinflamatório não hormonal & $17(74)$ & $8(80)$ \\
Metotrexate & 8 & 2 \\
Hidroxicloroquina & - & 2 \\
Prednisona & 5 & 6 \\
Abatacept & 2 & 5 \\
Azatioprina & - & 1 \\
\hline
\end{tabular}

Legenda: AIJ = artrite idiopática juvenil; ODAI = outras doenças auto-imunes $\mathrm{DP}=$ desvio-padrão

\section{Avaliação otológica e audiológica}

Durante a anamnese, 21 dentre os 48 avaliados apresentaram pelo menos uma queixa auditiva. Na Tabela 3 é apresentada a frequência das queixas.

A avaliação otológica evidenciou alteração de orelha média em $17 \%$ dos pacientes, os quais retornaram para a avaliação após o tratamento. Esses pacientes representavam 13\% do grupo controle, $17 \%$ do grupo AIJ e $20 \%$ do grupo ODAI. 
Tabela 3. Frequência de queixas auditivas nos Grupos Controle, Artrite Idiopática Juvenil e Outras Doenças Auto-imunes

\begin{tabular}{lcccc}
\hline Queixas & $\begin{array}{c}\text { Grupo Controle } \\
(\mathrm{n}=15)\end{array}$ & $\begin{array}{c}\text { Grupo AlJ } \\
(\mathrm{n}=23)\end{array}$ & $\begin{array}{c}\text { Grupo ODAl } \\
(\mathrm{n}=10)\end{array}$ & $\begin{array}{c}\text { Total } \\
(\mathrm{n}=48)\end{array}$ \\
\hline Zumbido & 1 & 7 & 1 & 11 \\
Otalgia & 1 & 5 & 2 & 7 \\
Vertigem & 2 & 2 & 1 & 5 \\
Secreção no ouvido & 3 & 1 & 0 & 3 \\
Diminuição da acuidade auditiva & 1 & 2 & 1 & 3 \\
Desatenção & 1 & 1 & 9 \\
\hline Total & 9 & 18 & \\
\hline
\end{tabular}

Qui-quadrado $x^{2}(p>0,05)$

Legenda: $\mathrm{AIJ}=$ artrite idiopática juvenil; ODAI = outras doenças auto-imunes

Pacientes dos grupos AIJ e ODAI apresentaram as mesmas queixas auditivas (zumbido, otalgia, vertigem, secreção no ouvido, diminuição da acuidade auditiva e desatenção). No inquérito de sintomas auditivos, $43 \%$ do grupo AIJ e $60 \%$ do grupo ODAI apresentaram pelo menos uma queixa auditiva. O zumbido foi a queixa mais frequente, seguido de otalgia e vertigem. Esses achados demonstram o ODAI como grupo com maior número de pacientes sintomáticos enquanto o AIJ apresentou maior proporção de sinais e sintomas auditivos.

Cada orelha foi analisada separadamente e na Tabela 4 é apresentada a frequência de alterações auditivas nos três grupos, em que verificam-se diferenças em todos os testes, mas a diferença significativa foi apenas nas emissões otoacústicas (Qui-quadrado p=0,004), com maior frequência de alterações em ambos os grupos AIJ e ODAI quando comparados ao grupo controle.

As alterações timpanométricas foram classificadas como "Ar", ou seja, evidenciaram rigidez de cadeia ossicular. A avaliação audiométrica apresentou o maior número de orelhas alteradas, classificadas como perda auditiva neurossensorial ${ }^{(26)}$ com limiares até $35 \mathrm{dBNA}$ em ambos os grupos AIJ e ODAI. No grupo AIJ foram observados comprometimentos nas frequências graves $(250,500 \mathrm{~Hz}$ e $1 \mathrm{kHz})$, médias ( 2 e $3 \mathrm{kHz}$ ) e agudas $(4,6$ e/ou $8 \mathrm{kHz})$, já no grupo ODAI apenas frequências graves e agudas mostraram alterações. As frequências agudas foram as mais alteradas no grupo AIJ e as graves no grupo ODAI.
Nos Potenciais Evocados Auditivos de Tronco Encefálico (PEATE) não foram observadas alterações em ambos os grupos, tendo sido observada a presença das ondas I, III e V; com morfologia e traçado adequados. Também na avaliação do Índice de Reconhecimento de Fala (IRF) todos os pacientes apresentaram resultados compatíveis com os padrões da normalidade.

\section{DISCUSSÃO}

A avaliação audiológica realizada foi a mais completa possível, no entanto, devido à baixa incidência e prevalência de doenças reumáticas pediátricas avaliou-se um número restrito de casos. Os resultados obtidos demonstraram que crianças e adolescentes com doenças reumáticas apresentam mais sintomas e alterações auditivas, quando comparadas a um grupo controle. A frequência de sintomas auditivos e achados da avaliação otológica e audiológica apontaram alterações importantes, assim como, em estudos precedentes realizados em adultos com as mesmas doenças reumáticas ${ }^{(1-10)}$.

Embora o número de alterações auditivas tenha sido maior entre os pacientes sintomáticos, foram observadas alterações em $46 \%$ dos pacientes assintomáticos do grupo AIJ e 50\% dos pacientes assintomáticos do grupo ODAI.

O zumbido é uma queixa comum em pacientes com doenças auto-imunes ${ }^{(1,10)}$. Esse sintoma, assim como a vertigem, esteve presente em nossos pacientes. O zumbido

Tabela 4. Frequência de resultados alterados em cada orelha dos pacientes dos grupos Controle, Artrite Idiopática Juvenil e Outras Doenças Auto-imunes

\begin{tabular}{lcccc}
\hline Teste & $\begin{array}{c}\text { Grupo Controle } \\
(\mathrm{n}=30)\end{array}$ & $\begin{array}{c}\text { Grupo AIJ } \\
(\mathrm{n}=46)\end{array}$ & $\begin{array}{c}\text { Grupo ODAl } \\
(\mathrm{n}=20)\end{array}$ & $\begin{array}{c}\text { Total } \\
(\mathrm{n}=96)\end{array}$ \\
\hline Timpanometria & 1 & 2 & 1 & 4 \\
Reflexo acústico & 0 & 7 & 2 & 9 \\
Audiometria & 6 & 16 & 7 & 29 \\
Emissões otoacústicas & 2 & 8 & $9^{*}$ & 19 \\
\hline
\end{tabular}

Qui-quadrado $x^{2}(p<0,05)$

* Controle x ODAI $\left(p=0,004 ; x^{2}=8,1634\right)$

AlJ $x$ ODAI $\left(p=0,04 ; x^{2}=4,2063\right)$

Legenda: AIJ = artrite idiopática juvenil; ODAI = outras doenças auto-imunes 
esteve presente em ambos os grupos AIJ e ODAI, mas não foi possível concluir se o zumbido poderia estar relacionado aos medicamentos em uso, por exemplo, os anti-inflamatórios não hormonais. A ocorrência de zumbidos poderia também ser explicada por alterações bioquímicas precedendo lesão anatômica.

O grupo AIJ apresentou maior número de alterações na timpanometria e pesquisa dos reflexos acústicos, podendo representar comprometimento de pequenas articulações da orelha média, resultando em queixas auditivas e alterações timpanométricas ${ }^{(11)}$.

As alterações timpanométricas encontradas não prejudicaram a captação das respostas nas emissões oto-acústicas (EOA). As orelhas que apresentaram curva tipo "Ar" na timpanometria tiveram EOA presentes. No entanto, as EOA também apontaram maior número de alterações nos grupos AIJ e ODAI, demonstrando um possível acometimento coclear ${ }^{(7,10)}$. O número de orelhas com alterações no teste de EOA foi maior do que no teste audiométrico, esse fato justifica-se pela sensibilidade do teste para detectar pequenas alterações nas células ciliadas externas, antes mesmo que elas sejam evidenciadas na audiometria. Esse fato pôde ser observado em um dos pacientes com esclerodermia localizada "en coup de sabre" afetando o lado direito da face, no qual a audiometria apresentou resultados normais enquanto as emissões otoacústicas estavam alteradas na orelha direita ${ }^{(16)}$.

Em nosso trabalho, observamos perda auditiva neurossensorial, assim como em diferentes estudos tanto em adultos ${ }^{(2,3,9,10,16)}$ como em crianças ${ }^{(12,13)}$. A audiometria mostrou alterações em todas as frequências, com limiares até $35 \mathrm{~dB}$, mas afetando principalmente as frequências agudas no grupo AIJ e as graves no grupo ODAI. O Índice de Reconhecimento de Fala apresentou resultados compatíveis com os limiares audiométricos em todos os pacientes avaliados. O resultado dos Potenciais Evocados de Tronco Encefálico descartaram qualquer envolvimento retrococlear, também descrito previamente em pacientes com doenças reumáticas ${ }^{(9)}$.

Pelo neurodiagnóstico acreditamos que o comprometimento auditivo dos pacientes avaliados seja de origem coclear. Observamos PEATE normais; com valores de latências absolutas e interpicos das ondas I, III e V dentro dos padrões da normalidade; mesmo nos pacientes que apresentaram audiometria e/ou EOA alterada. Procedimentos eletrofisiológicos atuais que avaliam com especificidade por frequência, assim como o PEATE utilizando-se como estímulo "tone burst" e ainda a Resposta Auditiva de Estado Estável (RAEE) não foram utilizados. Esses testes vão auxiliar no diagnóstico quando encontrarmos alterações no PEATE click $^{(27,28)}$ o que não foi o caso do nosso estudo.

Provavelmente, os exames de Longa Latência poderiam beneficiar as pesquisas com pacientes auto-imunes e detectar possíveis alterações no sistema nervoso auditivo central, uma vez que a fisiopatologia da doença poderia causar mudanças de origem funcional com diferentes manifestações em diferentes regiões cerebrais. No entanto, são realizados somente em pacientes acima de 12 anos, como nossa faixa etária era de 2 a 19 anos, esses testes não foram realizados.

Os testes utilizados na avaliação auditiva se complementam, apesar da avaliação das EOA serem mais sensíveis para detectar problemas cocleares, ela é restrita no que se refere às frequências avaliadas. Nenhum teste é completo o suficiente para realizar o diagnóstico auditivo de forma isolada. Portanto, a avaliação do sistema auditivo é complexa e pressupõe a utilização de procedimentos que devam ser interpretados em conjunto para obtenção de um diagnóstico seguro.

Entre as limitações deste estudo estão: a avaliação transversal, o pequeno número de sujeitos incluídos em uma amostra de conveniência atendida dentro do padrão de cuidados médicos. A duração média da doença foi dois anos e meio, podendo ter sido insuficiente para detectar os efeitos de dano cumulativo. Nestas doenças, os danos aos diversos órgãos e sistemas se acumulam com o tempo, assim como as consequências da intervenção terapêutica. Há ainda a possibilidade de que os mecanismos imunológicos envolvidos causem danos no aparelho auditivo de pacientes pediátricos de forma diversa dos adultos, o que justifica a avaliação sistemática.

O comprometimento auditivo pode-se relacionar aos diversos fatores como: mecanismos inflamatórios e auto-imunes, acometimento das pequenas articulações da orelha média, sensibilidade a alguns medicamentos como anti-inflamatórios e imunossupressores, incluindo os anti-inflamatórios não hormonais, prednisona, metotrexate, hidroxicloroquina, azatioprina e agentes biológicos. Por esse motivo, pode-se dizer que os mecanismos patogênicos e suas consequências ainda não foram totalmente elucidados, o que reforça a necessidade de estudos adicionais, controlando-se estas variáveis em estudos longitudinais.

\section{CONCLUSÃO}

Os sintomas relacionados à perda auditiva e as alterações audiológicas foram mais frequentes em crianças e adolescentes com Artrite Idiopática Juvenil e outras doenças auto-imunes. As alterações auditivas foram observadas também em pacientes assintomáticos, indicando a necessidade da avaliação auditiva sistemática como parte da rotina clínica desses pacientes.

\section{AGRADECIMENTOS}

Este trabalho foi desenvolvido como dissertação de Mestrado de Carolina Ferreira Campos-Flumian, com o auxílio da Fundação de Amparo à Pesquisa do Estado de São Paulo (FAPESP) (processo n ${ }^{\circ}$ 04/12950-4). Os autores agradecem a Ms. Josilene Luciene Duarte, Fonoaudióloga, pelo apoio na interpretação dos testes auditivos. 


\section{REFERÊNCIAS}

1. Stone JH, Francis HW. Immune-mediated inner ear disease. Curr Opin Rheumatol. 2000Jan;12(1):32-40.

2. Ruckenstein MJ. Autoimmune inner ear disease. Curr Opin Otolaryngol Head Neck Surg. 2004Oct;12(5):426-30.

3. Piccirillo JF. Steroids for idiopathic sudden sensorineural hearing loss: some questions answered, others remain. JAMA. 2011May;305(20):2114-5.

4. Kastanioudakis I, Ziavra N, Voulgari PV, Exarcharkos G, Skevas A, Drosos AA. Ear involvement in systemic lupus erythematosus patients: a comparative study. J Laryngol Otol 2002Feb;116(2):1037.

5. Iskandar SB, Loyd S, Roy TM. Cranial nerve VIII involvement in a patient with progressive systemic sclerosis. Tenn Med. 2004Mar;97(3):117-9.

6. Kastanioudakis I, Ziavra N, Politi EM, Exarchakos G, Drosos AA, Skevas A. Hearing loss in progressive systemic sclerosis patients: a comparative study. Otolaryngol Head Neck Surg. 2001May;124(5):522-5.

7. Ziavra N, Politi EN, Kastanioudakis I, Skevas A, Drosos AA. Hearing loss in Sjögren's syndrome patients. A comparative study. Clin Exp Rheumatol. 2000Nov-Dec;18(6):725-8.

8. Konttinen YT, Ramsay H, Hietanen J, Sorsa T, Nordstrom D. Otitis externa sicca/fibrotising external otitis (FEO) as complication of Sjögren's syndrome. Clin Exp Rheumatol. 2000NovDec:18(6):746-8.

9. Boki KA, Ioannidis JP, Segas JV, Maragkoudakis PV, Petrou D, Adamopoulos GK, et al. How significant is sensorineural hearing loss in primary Sjögren's syndrome? An individually matched casecontrol study. J Rheumatol. 2001Apr;28(4):798-801.

10. Papadimitraki ED, Kyrmizakis DE, Kritikos I, Boumpas DT. Earnose-throat manifestations of autoimmune rheumatic diseases. Clin Exp Rheumatol 2004Jul-Aug;22(4):485-94.

11. Giannini P, Marciano E, Saulino C, Strano CG, Alesio M, Marceli V, et al. Middle ear involvement in children with chronic rheumatoid juvenile arthritis. Eur Arch Otorhinolaryngol. 1997;254(Suppl.1):S30-3.

12. Berrettini S, Ravecca F, Sellari-Franceschini S, Matteucci F, Siciliano G, Ursino F. Progressive sensorineural hearing loss in childhood. Pediatr Neurol 1999Feb;20(2):130-6.

13. Tomasi JP, Lona A, Deggouj N, Gersdorff M. Autoimmune sensorineural hearing loss in young patients: an exploratory study. Laryngoscope 2001Nov;111(11Pt1):2050-3.

14. Ikis AO, Unsal E, Kirkim G, Erdag TK, Guneri EA. Hearing loss and middle ear involvement in patients with juvenile idiopathic arthitis. Int J Pediatr Otorhinolaryngol. 2007Jul;71(7):1079-85.

15. Sone M, Schachern PA, Paparella MM, Mirizono, N. Study of systemic lupus erythematosus in temporal bones. Ann Otol Rhinol Laryngol. 1999Apr;108(4):338-44.

16. Rauch SD, Halpin CF, Antonelli PJ, Babu S, Carey JP, Gantz BJ, et al. Oral vs intratympanic corticosteroid therapy for idiopathic sudden sensorineural hearing loss: a randomized trial. JAMA. 2011May;305(20):2071-9.

17. Saad-Magalhães C, Medeiros PBS, Sato JO, Domingues MAC. Clinical presentation and salivary gland histopathology of paediatric primary Sjögren's syndrome. Clinical Exp Rheumatol. 2011May;29(3):589-93.

18. Malleson PN, Connell H, Bennett SM, Eccleston C. Chronic musculoskeletal and other idiopathic pain syndromes. Arch Dis Child. 2001Mar;84(3):189-92.

19. Petty RE, Southwood TR, Manners P, Baum J, Glass DN, Goldenberg J, et al. International League of Associations for Rheumatology classification of juvenile idiopathic arthritis: second revision, Edmonton 2001. J Rheumatol. 2004Feb;31(2):390-2.

20. Hochberg MC. Updating the American College of Rheumatology revised criteria for the classification of systemic lupus erythematosus. Arthritis Rheum 1997Sep;40(9):1725.

21. Athreya BH. Juvenile Scleroderma. Curr Opin Rheumatol. 2002Sep;14(5):553-61.

22. Kassan SS, Moutsopoulos HM. Clinical manifestations and early diagnosis of Sjögren's syndrome. Arch Intern Med. 2004Jun;164(12):1275-84.

23. Jerger JF. Clinical experience with impedance audiometry. Arch Otolaryngol 1970Oct;92(4):311-24.

24. Roeser RJ. Manual de consulta rápida em audiologia: um guia prático. Rio de Janeiro: Revinter; 2001.

25. Jerger J, Speacks C, Trammell JL. A new approach to speech audiometry. J Speech Hear Disord. 1968Nov;33(4):318-28.

26. Silman S, Silverman CA. Basic audiologic testing. In: Silmann S, Silverman CA. Auditory diagnosis: principles and applications. San Diego: Singular Publishing Group, 1997; 44-52.

27. Duarte JL. A utilização da resposta auditiva de estado estável para estimar limiares auditivos em indivíduos com perda auditiva neurossensorial [dissertação]. Bauru: Universidade de São Paulo Faculdade de Odontologia de Bauru; 2007.

28. Linares AE. Correlação do potencial auditivo de estado estável com outros achados em audiologia pediátrica [tese]. São Paulo: Universidade de São Paulo - Faculdade de Medicina da Universidade de São Paulo; 2009. 